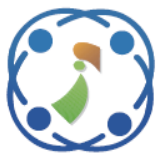

\title{
Performance of User Relaying Cooperative NOMA System Underlay Cognitive Radio Networks with Partial CSI
}

\author{
Nam-Soo Kim ${ }^{1 *}$ \\ ${ }^{I}$ Department of Electronic Engineering, Cheongju University, Republic of Korea \\ * Corresponding author's Email: nskim@cju.ac.kr
}

\begin{abstract}
In this paper, we study a cooperative non-orthogonal multiple access (NOMA) system underlying cognitive networks which adapt user relaying and selection combining. The selected relay among candidate near users is applied max relay selection rule utilizing partial channel state information (CSI). The outage probabilities of the user relay, far user, and the system are derived in closed-form, and conformed through Monte Carlo simulations. Numerical examples show that the outage probability of the user relay decreases with the tolerable interference power increases under the given conditions. Also, the outage probability of the far user decreases as the number of candidate relays increase. However, we noticed that when the tolerable interference-to-noise ratio exceeds $30 \mathrm{~dB}$ under given conditions, further performance improvements of the user relay and the far user does not achieve. Regarding the proposed system performance, it is noticed that the optimal power allocation coefficient, which minimize the outage probability, of the far user is approaching to 0.86 as the number of the candidate relays increase.
\end{abstract}

Keywords: NOMA, Cooperative NOMA, Cognitive radio, Partial CSI, User relay.

\section{Introduction}

Recent cellular mobile systems require higher spectral efficiency to satisfy the increasing traffic demand and spectrum scarcity. To satisfy the needs, non-orthogonal multiple access (NOMA) is proposed as a next generation multiple access [1-3]. Especially, a power domain NOMA system transmits multiple user's information simultaneously using same time and frequency. Accordingly, NOMA system can obtain the higher spectral efficiency than the conventional orthogonal multiple access (OMA) system which requires separate resources, i.e., time, frequency, and code. For this reason, NOMA has been received much attention in recent days.

In NOMA system, the more power is allocated to a far user to cope with the degradation caused in fading channels. The degradation can be improved with a cooperative communication, and as a result, the cell coverage extends $[4,5]$. During the process in successive interference cancellation (SIC) which removes the interferences from the multiplexed other users, the obtained information for the far user can be applied to cooperation in NOMA system. This cooperation is called user cooperative relaying. While the dedicated relaying, which is not utilize a user in NOMA system, requires a permanent standalone relay. The user cooperative relaying is more adequate for temporary networks such as AdHoc networks [6-9].

On the other hand, it is well known cognitive radio (CR) can increase spectral efficiency. There are two representative spectrum access methods; the opportunistic spectrum access (OSA) and underlay spectrum access (ULSA). A secondary user (SU) can utilize the space spectrum where primary users (PU) are not accessed in OSA [10, 11]. While in ULSA, the SU can share the spectrum simultaneously under the tolerable interference to PU $[12,13]$. Therefore the primary and secondary systems can be deployed in the same area, and can communicate their members without interruption.

More recently, cognitive NOMA systems which combine NOMA and CR have been studied for more efficient use of the scarce spectrum [14-18]. Y. Liu et el. considered NOMA in large-scale underlay CR 
networks with randomly deployed users [14]. However it did not include cooperative communication, relay selection and selection combining. L. Lv et al. considered a cooperative CR NOMA in which a base station transmits the superimposed signal of the primary and secondary user information [15]. It did not consider the power allocation between NOMA users which is an important system parameter in NOMA. In 2016, M. Zeng et al. analyzed the power allocation of superimposed signals to maximize the number of secondary users under the maximum tolerable interference to the primary receiver [16]. T. M. C. Chu et al. studied NOMA in CR networks with a dedicated relay, however, the user relay and relay selection did not include [17]. In [18], the resource allocation for video transmission in NOMA-based cognitive wireless networks was considered without cooperative communication.

Inspired by the above studies, a user cooperative NOMA system in underlay CR is considered in this paper. The user relay is selected among candidate near users utilizing partial channel state information (CSI) for the simple network configuration and performance improvement. Also, we adapt selection combing at the far user to achieve spatial diversity gain. The contributions of this paper are followings:

- Derive the performance of the user relay and a far user in a closed-form of a cooperative NOMA in CR underlay.

- Consider the effect of relay selection to the far user under the given tolerable interference to primary receiver.

- Analyze the effect of selection combining (S C) to NOMA in CR.

- Review the effect of power allocation to the performance of a NOMA system in CR.

The rest of this paper is organized as follows. The proposed user relaying cooperative NOMA system in $\mathrm{CR}$ underlay and relay selection process are described in Section 2. The outage probabilities of the user relay, far user, and the system are derived analytically in Section 3. In Section 4, numerical results are given and analyzed the obtained results. The analytical results are compared with the Monte Carlo simulations which confirm the analysis. Finally, the conclusions are given and future research topics are described in Section 5.

\section{System model}

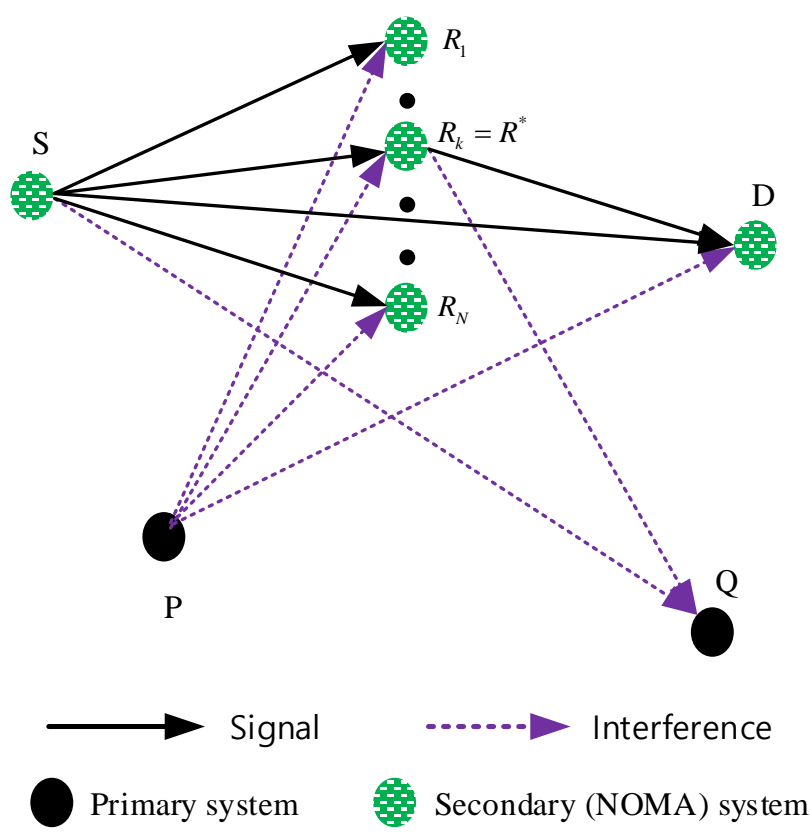

Figure.1 System model

The system model of the proposed cooperative NOMA underlay CR network is shown in Fig.1, which consist of a primary transmitter $(P)$, a primary receiver $(Q)$, a secondary transmitter $(S)$, a far user $(D)$, and $N$ near users $\left(R_{i}, i=1,2, \ldots, N\right)$. In this figure, the solid line and slotted line denote the signal and interference paths, respectively.

We assumed each channel has independent and identically distributed Rayleigh faded. The channel coefficient between node A and node B denotes $h_{A B}$. The cumulative distribution function (CDF) of $X_{A B}=\left|h_{A B}\right|^{2}$ is given by

$$
F_{X_{A B}}(x)=1-\exp \left(-\frac{x}{\Omega_{A B}}\right)
$$

and the probability density function (PDF) can be written by

$$
f_{X_{A B}}=\frac{1}{\Omega_{A B}} \exp \left(-\frac{x}{\Omega_{A B}}\right)
$$

where $\Omega_{A B}$ denotes the average channel power gain of $X_{A B}$.

The transmission protocol of the NOMA system has two phases, phase 1 and phase 2 . In phase $1, S$ broadcasts and $R_{i}$ and $D$ listen. The primary receiver $Q$ receives interference from $S$. Also $R_{i}$ receives the interference from the primary transmitter $P$.

In phase 2, the selected user among the near users, which decode the far user information successively, 
transmits to $D$. The selected user, a user relay, acts as a decode-and-forward (DF) relay. During the relay selection process, the partial CSI between $R_{i}$ and $D$ channels are employed. The user which has the best signal-to-noise ratio (SNR) of $R_{i}-D$ paths becomes the user relay. The index of the user relay can be denoted by

$$
k=\max _{i=1,2, \ldots, N}\left\{\gamma_{i D}\right\}
$$

where $\gamma_{i D}$ denoted the SNR of $R_{i}-D$ paths. For easy distinction, we denote the user relay $R_{k}$ to $R^{*}$.

The source $S$ in NOMA system transmits the multiplexed signal, which can be written by

$$
S_{T}=\sqrt{P_{S}}\left(\sqrt{\alpha_{R}} x_{R}+\sqrt{\alpha_{D}} x_{D}\right)
$$

where $X_{R}$ and $X_{D}$ denote the information to near users and $D$, respectively, with $\left|x_{R}\right|^{2}=\left|x_{D}\right|^{2}=1 \cdot \alpha_{R}$ and $\alpha_{D}$ denote the power allocation coefficient of the near user and $D$, respectively, with $\alpha_{R}+\alpha_{D}=1$, $\alpha_{R} \leq \alpha_{D} \cdot P_{S}$ denotes the transmission power of $S$, which takes the minimum value between the tolerable interference of the primary receiver $Q$ or the maximum transmission power of $S$,

$$
P_{S}=\min \left(\frac{I}{\left|h_{S Q}\right|^{2}}, P_{1}\right)
$$

where $I$ denotes the tolerable interference of the primary receiver, and $P_{1}$ denotes the maximum transmission power of $S$.

The received signal at $i$-th near user $R_{i}$ can be written by

$$
y_{R_{i}}=h_{S i} \sqrt{P_{S}}\left(\sqrt{\alpha_{R}} x_{R}+\sqrt{\alpha_{D}} x_{D}\right)+n_{i}
$$

where $n_{i}$ denotes noise at $R_{i}$ plus interference from $P$, which is Gaussian distributed and $n_{i} \sim$ $N\left(0, N_{0}+P_{T} d_{P i}^{-n}\right)$ [14]. And where $N_{0}$ is the noise power of $R_{i}, P_{T}$ denotes the transmission power of $P$, $n$ denotes the path loss exponent which has 3 in urban area. $d_{A B}$ is the distance between node $A$ and $B$.

The signal-to-interference plus noise ratio (SINR) of $R_{i}$ for decoding $X_{D}$ can be written by

$$
\gamma_{S i}^{\chi_{D}}=\frac{\left|h_{S i}\right|^{2} P_{S} \alpha_{D}}{\left|h_{S i}\right|^{2} P_{S} \alpha_{R}+N_{i}}
$$

where $N_{i}=N_{0}+P_{T} d_{P i}^{-n}$. Therefore, by replacing $i$ to $R^{*}$ and apply Eq. (5) into Eq. (7), we can write the SINR of $R^{*}$,

$$
\gamma_{S R^{*}}^{X_{D}}=\frac{X_{S R^{*}} \theta_{1}}{X_{S R^{*}}+1 / Z}
$$

where $\theta_{1}=\alpha_{D} / \alpha_{R}$, and

$$
Z=\min \left(\frac{\theta_{2}}{X_{S Q}}, \theta_{3}\right)
$$

where $\theta_{2}=\alpha_{R} I / N_{R^{*}} \quad, \quad \theta_{3}=\alpha_{R} P_{1} / N_{R^{*}} \quad, \quad$ and $N_{R^{*}}=N_{0}+P_{T} d_{P R^{*}}^{-n}$

The interference from the multiplexed other user signals are removed by SIC in power domain NOMA system. After SIC processing, SNR of $R_{i}$ for decoding $X_{R}$ can be written by

$$
\gamma_{S i}^{x_{R}}=\frac{\left|h_{S i}\right|^{2} P_{S} \alpha_{R}}{N_{i}} .
$$

Replacing Eq. (5) into Eq. (10), we can rearrange SNR of $R^{*}$ for decoding $X_{R}$ can be obtained by

$\gamma_{S R^{*}}^{x_{R}}=X_{S R^{*}} Z$

The received signal at $D$ from $R^{*}-D$ path is given by

$y_{R^{*} D}=h_{R^{*} D} \sqrt{P_{R^{*}}} x_{D}+n_{D}$

where $n_{D}$ denotes noise at $D$ plus interference from $P$, which is Gaussian distributed and $n_{D} \sim$ $N\left(0, N_{0}+P_{T} d_{P D}^{-n}\right)$. And $P_{R^{*}}$ denotes the transmission power of $R^{*}$,

$P_{R^{*}}=\min \left(\frac{I}{\left|h_{R^{*} Q}\right|^{2}}, P_{2}\right)$

where $P_{2}$ is the maximum transmission power of $R^{*}$. Notice that the transmission time of $S$ and $R^{*}$ is different in phase 1 and phase 2 , respectively, hence, the tolerable interference of the primary receiver $Q$ is identical to $I$ irrespective of $S$ or $R^{*}$ transmission. The SINR at $D$ from $R^{*}-D$ path can be written by 


$$
\gamma_{R^{*} D}^{x_{D}}=\frac{\left|h_{R^{*} D}\right|^{2} P_{R^{*}}}{N_{D}}=X_{R^{*} D} Y
$$

where

$Y=\min \left(\frac{\theta_{4}}{X_{R^{*} Q}}, \theta_{5}\right)$

and where $\theta_{4}=\alpha_{D} I / N_{D}$, and $\theta_{5}=\alpha_{D} P_{2} / N_{D}$.

The received signal at $D$ from the direct path of $S-D$ is given by

$y_{S D}=h_{S D} \sqrt{P_{S}}\left(\sqrt{\alpha_{R}} x_{R}+\sqrt{\alpha_{D}} x_{D}\right)+n_{D}$

The SNR from the direct path can be written by

$\gamma_{S D}^{x_{D}}=\frac{\left|h_{S D}\right|^{2} \alpha_{D}}{\left|h_{S D}\right|^{2} \alpha_{R}+N_{D} / P_{S}}=\frac{X_{S D} \theta_{1}}{X_{S D}+1 / W}$

where

$W=\min \left(\frac{\theta_{6}}{X_{\mathrm{SQ}}}, \theta_{7}\right)$

and $\theta_{6}=\alpha_{R} I / N_{D}, \theta_{1}=\alpha_{R} P_{1} / N_{D}$.

\section{Outage probability}

Since the information for $R^{*}$ is different from $D$, the outage probability of $R^{*}$ and $D$ is derived respectively. Also the system outage probability of $R^{*}$ and $D$ paired is derived.

\subsection{Outage probability of $R^{*}$}

An outage event happens following one of the cases; when the SINR of $D$ for $X_{D}$ at $R^{*}$ less than the threshold, therefore $X_{D}$ cannot be decoded. Next, though $X_{D}$ decoded successfully, the SNR of $R^{*}$ for $X_{R}$ after SIC cannot be decoded. Therefore, the outage probability of $R^{*}$ can be written by

$$
P_{o, R^{*}}=\operatorname{Pr}\left(\gamma_{S R^{*}}^{x_{D}}<\Gamma_{D}\right)+\operatorname{Pr}\left(\gamma_{S R^{*}}^{x_{D}} \geq \Gamma_{D}, \gamma_{S R^{*}}^{x_{R}}<\Gamma_{R}\right)
$$

where $\Gamma_{D}=2^{2 R_{D}}-1$, and $\Gamma_{R}=2^{R_{R}}-1 \cdot R_{D}$ and $R_{R}$ denote the required spectral efficiency [bps/Hz] of $D$ and $R^{*}$, respectively. The first probability $\operatorname{Pr}\left(\gamma_{S R^{*}}^{x_{D}}<\Gamma_{D}\right)$ of Eq. (19) can be rearranged by replacing $\gamma_{S R^{*}}^{x_{D}}$ with Eq.
$\operatorname{Pr}\left(\gamma_{S R^{*}}^{x_{D}}<\Gamma_{D}\right)=\operatorname{Pr}\left(Z<\frac{\Gamma_{D}}{X_{S R^{*}}\left(\theta_{1}-\Gamma_{D}\right)}\right)=F_{Z}\left(\frac{\Gamma_{D}}{X_{S R^{*}}\left(\theta_{1}-\Gamma_{D}\right)}\right)$

Apply Eq. (11) into the second probability, $\operatorname{Pr}\left(\gamma_{S R^{*}}^{x_{R}}<\Gamma_{R}\right)$ of Eq. (19), we can write

$\operatorname{Pr}\left(\gamma_{S R^{*}}^{x_{R}}<\Gamma_{R}\right)=\operatorname{Pr}\left(Z<\frac{\Gamma_{R}}{X_{S R^{*}}}\right)=F_{Z}\left(\frac{\Gamma_{R}}{X_{S R^{*}}}\right)$.

By replacing Eqs. (20) and (21) into Eq. (19), we can rewrite

$$
\begin{aligned}
P_{o, R^{*}} & =\operatorname{Pr}\left(Z<\frac{\Gamma_{D}}{X_{S R^{*}}\left(\theta_{1}-\Gamma_{D}\right)}\right) \\
& +\operatorname{Pr}\left(Z \geq \frac{\Gamma_{D}}{X_{S R^{*}}\left(\theta_{1}-\Gamma_{D}\right)}, Z<\frac{\Gamma_{R}}{X_{S R^{*}}}\right)
\end{aligned}
$$

Eq. (22) can be rearranged by

$$
\begin{aligned}
P_{o, R^{*}}= & \begin{cases}\operatorname{Pr}\left(Z<\frac{\Gamma_{D}}{X_{S R^{*}}\left(\theta_{1}-\Gamma_{D}\right)}\right), & \Gamma_{R}<\frac{\Gamma_{D}}{\left(\theta_{1}-\Gamma_{D}\right)} \\
\operatorname{Pr}\left(Z<\frac{\Gamma_{R}}{X_{S R^{*}}}\right), & \Gamma_{R} \geq \frac{\Gamma_{D}}{\left(\theta_{1}-\Gamma_{D}\right)}\end{cases} \\
= & F_{Z}\left\{\max \left(\frac{\Gamma_{D}}{X_{S R^{*}}\left(\theta_{1}-\Gamma_{D}\right)}, \frac{\Gamma_{R}}{X_{S R^{*}}}\right)\right\}
\end{aligned}
$$

where $\max (a, b)$ takes the maximum value between $a$ or $b$.

The outage probability of $R^{*}$ can be written by Eq. (24) on the next page (see Appendix).

\subsection{Outage probability of $D$}

We assumed $D$ combine the received signals both from the indirect path $\left(R^{*}-D\right.$ path) and the direct path ( $S-D$ path) by SC. An outage event happens when both paths fail. Since two paths are independent, the outage probability can be written by

$P_{o, D}=P_{o, R^{*} D} \times P_{o, S D}$

where $P_{O, R^{*} D}$ and $P_{O, S D}$ denote outage probability of $R^{*}-D$ and $S-D$ path, respectively.

Firstly, consider the outage probability of indirect path of $R^{*}-D, P_{O, R^{*} D}$. Since the selected near user among the successively decoded near users acts as a DF relay, the outage probability of $D$ can be written by

(8), 


$$
P_{o, R^{*} D}=\sum_{i=0}^{N} \operatorname{Pr}\left(\gamma_{R^{*} D}^{x_{D}}<\Gamma_{D}|R(s)|=i\right) \operatorname{Pr}(|R(s)|=i)
$$

where $|R(s)|$ is the number of the successively decoded near users of $S-R_{i}(i=1,2, \ldots, N)$ paths. $\operatorname{Pr}\left(\gamma_{R^{*} D}^{x_{D}}<\Gamma_{D}\right)$ in Eq. (26) can be obtained from Eqs. (14) and (15),

$\operatorname{Pr}\left(\gamma_{R^{*} D}^{x_{D}}<\Gamma_{D}\right)=\operatorname{Pr}\left(Y<\frac{\Gamma_{D}}{X_{R^{*} D}}\right)=F_{Y}\left(\frac{\Gamma_{D}}{X_{R^{*} D}}\right)$.

By replacing Eq. (15) with $Y$, Eq. (27) can be rearranged by

$F_{Y}\left(\frac{\Gamma_{D}}{X_{R^{*} D}}\right)=\left\{\begin{array}{ll}1, & X_{R^{*} D} \leq \frac{\Gamma_{D}}{\theta_{5}} \\ \exp \left(-\frac{\theta_{4}}{\Gamma_{D} \Omega_{R^{*} D}} X_{R^{* D}}\right), & X_{R^{*} D}>\frac{\Gamma_{D}}{\theta_{5}}\end{array}\right.$.

Similar to (A.6), $Y$ and $X_{R^{*} D}$ are random variable in Eq. (28), we can average the CDF

$F_{Y}\left(\frac{\Gamma_{D}}{X_{R^{*} D}}\right)=\int_{0}^{\infty} F_{Y}\left(\frac{\Gamma_{D}}{X_{R^{*} D}}\right) f_{R^{*} D}\left(x_{R^{*} D}\right) d x_{R^{*} D}$.
Therefore, replace $X_{S R^{*}}$ in Eq. (28) with $x_{S R^{*}}$, then the CDF of $F_{Y}\left(\frac{\Gamma_{D}}{x_{R^{*} D}}\right)$ can be written by Eq. (30). $f_{X_{R^{*} D}}\left(x_{R^{*} D}\right)$ is the pdf of $R^{*}$ in Rayleigh fading, which can be written by

$$
\begin{aligned}
f_{X_{R^{*} D}}\left(x_{R^{*} D}\right) & =\frac{i}{\Omega_{R^{*} D}}\left(1-e^{-X_{R^{*} D} / \Omega_{R^{*} D}}\right)^{i-1} e^{-X_{R^{*} D} / \Omega_{R^{*} D}}, \\
& =\sum_{l=1}^{i}\left(\begin{array}{l}
i \\
I
\end{array}\right)(-1)^{-i} \frac{i}{\Omega_{R^{*} D}} \exp \left(-i X_{R^{*} D} / \Omega_{R^{*} D}\right)
\end{aligned}
$$

where the second equality holds from the binomial expansion. And the CDF is given by

$$
F_{X_{R^{*} D}}\left(X_{R^{*} D}\right)=\left(1-e^{-X_{R^{*} D} / \Omega_{R^{*} D}}\right)^{i}
$$

where $i$ represents the number of $R(s) \mid$ in Eq. (26). By replacing Eq. (31) into Eq. (30), Eq. (27) can be rewritten by Eq. (33).

Meanwhile, the second probability in Eq. (26) can be represented by

$$
\operatorname{Pr}(|R(s)|=i)=\left(\begin{array}{l}
N \\
i
\end{array}\right)\left(1-P_{o, R^{*}}\right) P_{o, R^{*}}^{N-i}
$$

$$
P_{o, R^{*}}= \begin{cases}1-\exp \left(-\frac{\Gamma_{D}}{\Omega_{S R^{*}}\left(\theta_{1}-\Gamma_{D}\right) \theta_{3}}\right)+\frac{\Omega_{S D} \Gamma_{D}}{\Omega_{S R^{*}}\left(\theta_{1}-\Gamma_{D}\right) \theta_{2}+\Omega_{S Q} \Gamma_{D}} \exp \left\{-\left(\frac{\theta_{2}}{\Omega_{S Q} \theta_{3}}+\frac{\Gamma_{D}}{\Omega_{S R^{*}}\left(\theta_{1}-\Gamma_{D}\right) \theta_{3}}\right)\right\}, \Gamma_{R}<\Gamma_{D} /\left(\theta_{1}-\Gamma_{D}\right) \\ 1-\exp \left(-\frac{\Gamma_{R}}{\Omega_{S R^{*}} \theta_{3}}\right)+\frac{\Omega_{S Q} \Gamma_{R}}{\Omega_{S R^{*}} \theta_{2}+\Omega_{S Q} \Gamma_{R}} \exp \left\{-\left(\frac{\theta_{2}}{\Omega_{S Q} \Gamma_{R}}+\frac{1}{\Omega_{S R^{*}}}\right) \frac{\Gamma_{R}}{\theta_{3}}\right\}, & \Gamma_{R} \geq \Gamma_{D} /\left(\theta_{1}-\Gamma_{D}\right)\end{cases}
$$

$$
F_{Y}\left(\frac{\Gamma_{D}}{X_{R^{*} D}}\right)=\int_{0}^{\Gamma_{D} / \theta_{5}} f_{X_{R^{*} D}}\left(x_{R^{*} D}\right) d x_{R^{*} D}+\int_{\Gamma_{D} / \theta_{5}}^{\infty} \exp \left(-\frac{\theta_{4}}{\Gamma_{D} \Omega_{R^{*} D}} x_{R^{*} D}\right) f x_{R^{*} D}\left(x_{R^{*} D}\right) d x_{R^{*} D}
$$

$$
\operatorname{Pr}\left(\gamma_{R^{*} D}^{x_{D}}<\Gamma_{D}\right)=\left\{1-\exp \left(-\frac{\Gamma_{D}}{\theta_{5} \Omega_{R^{*} D}}\right)\right\}^{i}+\sum_{l=1}^{i}\left(\begin{array}{l}
i \\
l
\end{array}\right)(-1)^{\gamma-1} \frac{1 \Omega_{R^{*} Q} \Gamma_{D}}{\theta_{4} \Omega_{R^{*} D}+\Omega_{R^{*} Q} \Gamma_{D}} \exp \left\{-\left(\frac{\theta_{4}}{\Omega_{R^{*} Q} \Gamma_{D}}+\frac{i}{\Omega_{R^{*} D}}\right) \frac{\Gamma_{D}}{\theta_{5}}\right\}
$$


where $P_{o, R^{*}}$ is the outage probability of $R^{*}$ given in Eq. (24). Therefore, the outage probability of the indirect path given in Eq. (26) can be obtained from Eqs. (33) and (34), and given by Eq. (35).

Secondly, consider the outage probability of the direct path. An outage happens the received SINR of $D$ from $S-D$ path bellows the threshold, and the outage probability of the direct path $P_{O, S D}$ can be obtained by applying Eq. (17)

$$
\begin{aligned}
P_{O, S D} & =\operatorname{Pr}\left(\gamma_{S D}^{x_{D}}<\Gamma_{D}\right)=\operatorname{Pr}\left(W<\frac{\Gamma_{D}}{X_{S D}\left(\theta_{1}-\Gamma_{D}\right)}\right) . \\
& =F_{W}\left(\frac{\Gamma_{D}}{X_{S D}\left(\theta_{1}-\Gamma_{D}\right)}\right)
\end{aligned}
$$

Similarly, replacing Eq. (18) into Eq. (36), Eq. (36) can be written by

$F_{W}\left(\frac{\Gamma_{D}}{X_{S D}\left(\theta_{1}-\Gamma_{D}\right)}\right)=\left\{\begin{array}{ll}1, & X_{S D} \leq \frac{\Gamma_{D}}{\left(\theta_{1}-\Gamma_{D}\right) \theta_{7}} . \\ \exp \left(-\frac{\theta_{6}}{\Omega_{S Q}} W\right), & X_{S D}>\frac{\Gamma_{D}}{\left(\theta_{1}-\Gamma_{D}\right) \theta_{7}}\end{array}\right.$.

And by taking average, it becomes

$$
F_{W}\left(\frac{\Gamma_{D}}{x_{S D}\left(\theta_{1}-\Gamma_{D}\right)}\right)=\int_{0}^{\infty} F_{W}\left(\frac{\Gamma_{D}}{x_{S D}\left(\theta_{1}-\Gamma_{D}\right)}\right) x_{S D}\left(x_{S D}\right) d x_{S D} \cdot
$$

From Eqs. (37) and (38), the outage probability of the direct path given in Eq. (36) can be written by

$$
\begin{aligned}
& P_{o, S D}=1-\exp \left(-\frac{\Gamma_{D}}{\Omega_{S D}\left(\theta_{1}-\Gamma_{D}\right) \theta_{7}}\right) \\
& +\frac{\Omega_{S Q} \Gamma_{D}}{\theta_{6}\left(\theta_{1}-\Gamma_{D}\right) \Omega_{S D}+\Omega_{S Q} \Gamma_{D}} \exp \left\{-\left(\frac{\theta_{6}}{\Omega_{S Q} \theta_{7}}+\frac{\Gamma_{D}}{\Omega_{S D}\left(\theta_{1}-\Gamma_{D}\right) \theta_{7}}\right)\right\}
\end{aligned}
$$

By replacing Eqs. (35) and (39) into Eq. (25), we can obtain the outage probability of $D$.

\subsection{Outage probability of cooperative NOMA system in CR}

The outage of the proposed NOMA system is happens either $R^{*}$ or $D$ is in outage. Hence outage

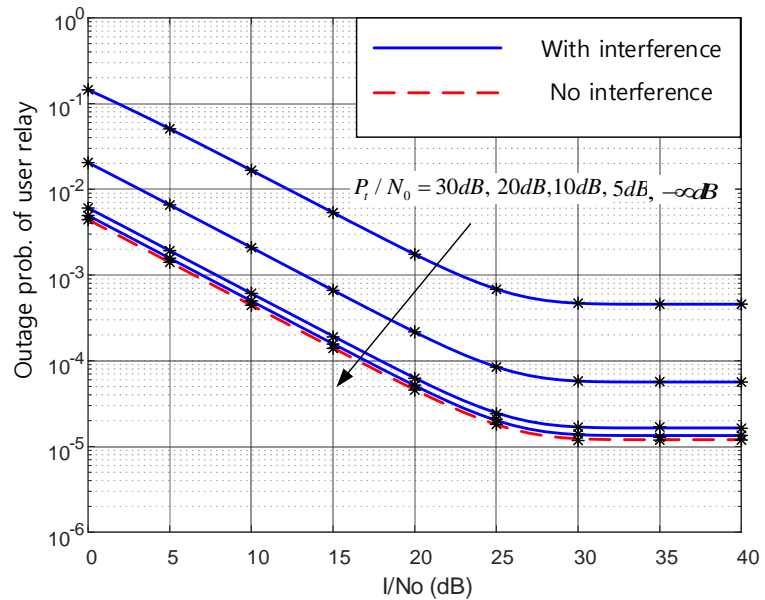

Figure. 2 Outage probability of user relay $R^{*}$

probability of the proposed NOMA system can be defined by

$$
\begin{aligned}
P_{o, s y s} & =1-P_{\text {succ }} \\
& =1-\left(1-P_{o, R^{*}}\right)\left(1-P_{o, D}\right)
\end{aligned}
$$

It can be obtained from Eqs. (24) and (25), in conjunction with Eqs. (35) and (39).

\section{Numerical examples}

In this section, numerical and simulation results are shown as a function of the tolerable interference to the primary receiver. Also the effect of the power allocation coefficient to the outage probability is demonstrated. The system parameters for the numerical example are selected as $R_{F}=R_{R}=1$, $P_{1} / N_{0}=40 d B, P_{2} / N_{0}=10 d B, n=3, \alpha_{D}=0.8$, and $\alpha_{R}=0.2$. Also the normalized distances of the links are selected as $d_{S R^{*}}=d_{S R_{i}}=0.2, d_{R^{*} D}=d_{R_{i} D}=0.8$, $d_{S D}=1, d_{S Q}=3, d_{P R^{*}}=3, d_{P D}=3, d_{P Q}=2$.

In Fig. 2, the solid lines denote the outage probability of the user relay $R^{*}$ with different kinds of interference from the primary transmitter. As the transmission power of the primary transmitter decreases, the outage probability of the user relay is approaching to the slotted line curve which denotes the outage probability of $R^{*}$ without interference from the primary transmitter (i.e., $P_{T}=0$ in Eq. (9) and Eq. (12)). We can notice the effect of the interference to the outage probability is negligible up to $10 \mathrm{~dB}$, however, significant over $20 \mathrm{~dB}$ of $P_{t} / N_{0}$.

As the tolerable interference-to-noise ratio $\left(I / N_{0}\right)$ exceeds $30 \mathrm{~dB}$, the outage probability floor is noticed. 


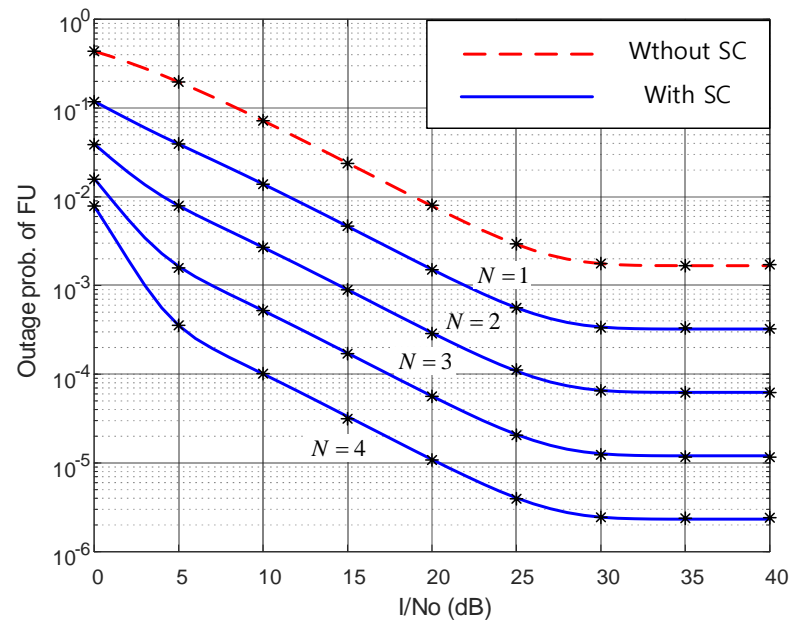

Figure. 3 Outage probability of far user $D\left(P_{t} / N_{0}=5 d B\right)$

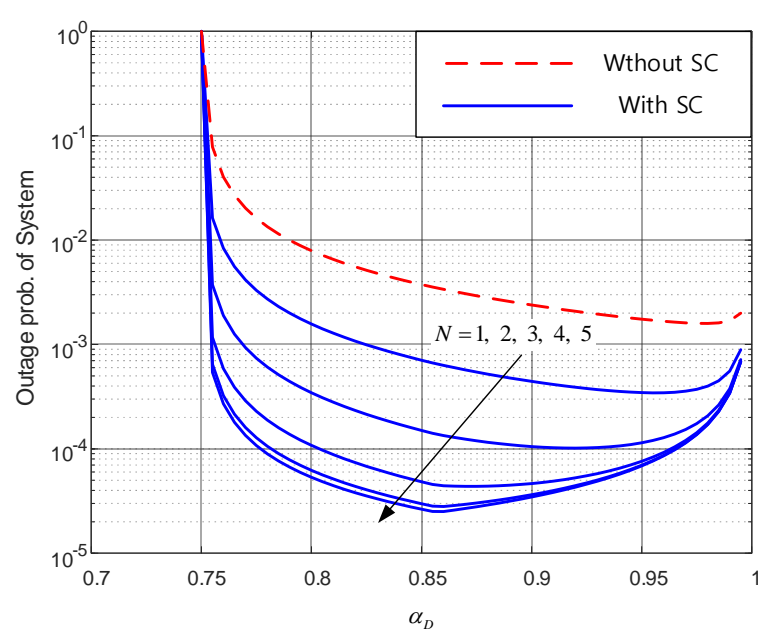

Figure. 4 Outage probability of the proposed NOMA $\operatorname{system}\left(P_{t} / N_{0}=5 d B, I / N_{0}=20 d B\right)$

It is interpreted that when $I / N_{0}$ exceeds $30 \mathrm{~dB}, S$ in NOMA system transmits its maximum power. The transmission power is no longer increase, hence, the performance does not improve. In this figure, “*” represent the Monte Carlo simulation results which perfectly match with the analytical results. It reveals the analytically derived results are accurate.

The outage probability of $D$ is shown in Fig.3, which indicates the performance improves with the number of candidate relays. Also the slotted line shows the outage probability of the direct path $(S-D$ path) only in Eq. (39), which means no SC. It is observed that the difference of $I / N_{0}$ is about $7 \mathrm{~dB}$ compared with $N=1$ for outage probability of $1 \times 10^{-2}$. From this figure we noticed that even in CR, the cooperative NOMA with SC always has better performance than without SC.

As the number of candidate relays increase, the tolerable $I / N_{0}$ reduces about $7.5 \mathrm{~dB}$ to maintain the identical outage probability between $5 \mathrm{~dB}$ to $25 \mathrm{~dB}$ of
$I / N_{0}$. Similar to Fig. 2, beyond $30 \mathrm{~dB}$ of $I / N_{0}$, the outage probability of $D$ is constant in Fig. 3. Since when $I / N_{0}$ exceeds $30 \mathrm{~dB}, R^{*}$ in NOMA system transmits its maximum power. Hence, further improvement of outage probability is not expected.

As mentioned in Fig. 2, “*” represent the Monte Carlo simulation results. It is shown that the simulation and the analytical results are perfectly matched, which revels the analytically derived results are accurate.

The outage probability of the proposed system versus power allocation coefficient to $D$ is shown in Fig. 4. The outage probability of the system incurred by the outage probability of $D$ and $R^{*}$. As the power allocation to $D$ increases, the outage probability of $D$ decreases, while, that of $R^{*}$ has concave curve. Therefore, the outage probability of the system shows concave in Fig. 4. It means the optimal power allocation coefficient which minimize the outage probability exists.

The optimal power allocation coefficient of $\alpha_{D}$ is $0.96,0.92,0.87,0.86,0.86$ as the number of candidate relays increase from 1 to 5 , respectively.

We noticed that the optimal power allocation coefficient under the given condition is approaching to 0.86 with the number of the candidate relays. The slotted line in Fig. 4 indicates the outage probability of the direct path only in Eq. (39), without the indirect path, which shows the optimal power allocation coefficient has 0.98 .

\section{Conclusions}

In this paper, the outage probability of a user relaying cooperative NOMA system underlay $\mathrm{CR}$ is derived analytically. We consider a user relaying instead of a dedicated relaying for simple network configuration, and apply max relay selection to improve the performance. Further, selection combing is adapted for the special diversity gain. The derived results are validated with Monte Carlo simulation.

Numerical examples show that the outage probability of the user relay decreases as the transmission power of the primary transmitter decreases and the tolerable interference power increases. However, when $I / N_{0}$ exceeds $30 \mathrm{~dB}$ under the given conditions, the source of NOMA system transmits its maximum power, hence, more performance improvements do not achieved.

On the other hands, in the range between $5 \mathrm{~dB}$ and $30 \mathrm{~dB}$ of $I / N_{0}$, the outage probability of the far user decreases as the number of candidate relays increase. Similarly, above $30 \mathrm{~dB}$ of $I / N_{0}$, the user relay 
transmits its maximum power and further performance improvements of the far user do not observed.

As expected, the system performance improves with the number of the candidate relays. It is noticed that an optimal power allocation for minimizing the outage probability is expected. Under the given condition, the optimal power allocation coefficient of the far user is approaching to 0.86 with the number of the candidate relays increases.

Further research will be focused on the performance analysis in various fading channel models with different kinds of relay selection protocols.

\section{Appendix}

Consider the CDF of $z, F_{z}(z)$, can be obtained by substituting $z$ by Eq. (9), and written by

$$
F_{Z}(z)=\operatorname{Pr}\left\{\min \left(\frac{\theta_{2}}{X_{S Q}}, \theta_{3}\right)<z\right\}
$$

If $\theta_{2} / X_{S Q} \geq \theta_{3}$, (A.1) becomes

$$
\begin{aligned}
F_{Z}(z) & =\operatorname{Pr}\left(\theta_{3}<z\right) \\
& =1, \quad z>\theta_{3}
\end{aligned}
$$

Otherwise, if $\theta_{2} / X_{S Q}<\theta_{3}$,

$$
\begin{aligned}
F_{Z}(z) & =\operatorname{Pr}\left(\frac{\theta_{2}}{X_{S Q}}<z\right)=\operatorname{Pr}\left(X_{S Q}>\frac{\theta_{2}}{z}\right) . \\
& =1-F_{X_{S Q}}\left(\frac{\theta_{2}}{z}\right), \quad z \leq \theta_{3}
\end{aligned}
$$

$$
\begin{aligned}
& F_{Z}\left\{\max \left(\frac{\Gamma_{D}}{X_{S R^{*}}\left(\theta_{1}-\Gamma_{D}\right)}, \frac{\Gamma_{R}}{X_{S R^{*}}}\right)\right\}=F_{Z}\left(\frac{\Gamma_{R}}{X_{S R^{*}}}\right)=\operatorname{Pr}\left\{\min \left(\frac{\theta_{2}}{X_{S Q}}, \theta_{3}\right)<\frac{\Gamma_{R}}{X_{S R^{*}}}\right\} \\
& = \begin{cases}1, & X_{S R^{*}} \leq \frac{\Gamma_{R}}{\theta_{3}} \\
\exp \left(-\frac{\theta_{2}}{\Omega_{S Q}} \frac{x_{S R^{*}}}{\Gamma_{R}}\right), & X_{S R^{*}}>\frac{\Gamma_{R}}{\theta_{3}}\end{cases}
\end{aligned}
$$

$$
\begin{aligned}
& F_{Z}\left\{\max \left(\frac{\Gamma_{D}}{X_{S R^{*}}\left(\theta_{1}-\Gamma_{D}\right)}, \frac{\Gamma_{R}}{X_{S R^{*}}}\right)\right\}=F_{Z}\left(\frac{\Gamma_{D}}{X_{S R^{*}}\left(\theta_{1}-\Gamma_{D}\right)}\right)=\operatorname{Pr}\left\{\min \left(\frac{\theta_{2}}{X_{S Q}}, \theta_{3}\right)<\frac{\Gamma_{D}}{X_{S R^{*}}\left(\theta_{1}-\Gamma_{D}\right)}\right\} \\
& = \begin{cases}1, & X_{S R^{*}} \leq \frac{\Gamma_{D}}{\theta_{3}\left(\theta_{1}-\Gamma_{D}\right)} \\
\exp \left(-\frac{\theta_{2}}{\Omega_{S Q}} \frac{\theta_{1}-\Gamma_{D}}{\Gamma_{D}} x_{S R^{*}}\right), & X_{S R^{*}}>\frac{\Gamma_{D}}{\theta_{3}\left(\theta_{1}-\Gamma_{D}\right)}\end{cases}
\end{aligned}
$$

$$
\begin{aligned}
P_{o, R^{*}} & =\int_{0}^{\varepsilon} f_{X_{S R^{*}}}\left(x_{S R^{*}}\right) d x_{S R^{*}}+\int_{\varepsilon}^{\infty} \exp \left(-\frac{\theta_{2}}{\Omega_{S Q}} \frac{\theta_{1}-\Gamma_{D}}{\Gamma_{D}} x_{S R^{*}}\right) f_{S R^{*}}\left(x_{S R^{*}}\right) d x_{S R^{*}} \\
& =1-\exp \left(-\frac{\Gamma_{D}}{\Omega_{S R^{*}}\left(\theta_{1}-\Gamma_{D}\right) \theta_{3}}\right)+\frac{\Omega_{S Q} \Gamma_{D}}{\Omega_{S R^{*}}\left(\theta_{1}-\Gamma_{D}\right) \theta_{2}+\Omega_{S Q} \Gamma_{D}} \exp \left\{-\left(\frac{\theta_{2}}{\Omega_{S Q} \theta_{3}}+\frac{\Gamma_{D}}{\Omega_{S R^{*}}\left(\theta_{1}-\Gamma_{D}\right) \theta_{3}}\right)\right\}, \Gamma_{R}<\Gamma_{D} /\left(\theta_{1}-\Gamma_{D}\right)
\end{aligned}
$$

By applying (A.2) and (A.3) into Eq. (23), if $\Gamma_{R}<\Gamma_{D} /\left(\theta_{1}-\Gamma_{D}\right)$, Eq. (23) can be rearranged by (A.4). Otherwise, if $\Gamma_{R} \geq \Gamma_{D} /\left(\theta_{1}-\Gamma_{D}\right)$, Eq. (23) becomes (A.5). The last equality of (A.4) and (A.5) are applied Rayleigh fading. 


$$
P_{o, R^{*}}=\int_{0}^{\infty} F_{Z}\left\{\max \left(\frac{\Gamma_{D}}{x_{S R^{*}}\left(\theta_{1}-\Gamma_{D}\right)}, \frac{\Gamma_{R}}{x_{S R^{*}}}\right)\right\} f_{x_{S R^{*}}}\left(x_{S R^{*}}\right) d x_{S R^{*}}
$$

Therefore, the CDF of $F_{Z}\left\{\max \left(\frac{\Gamma_{D}}{x_{S R^{*}}\left(\theta_{1}-\Gamma_{D}\right)}, \frac{\Gamma_{R}}{x_{S R^{*}}}\right)\right\}$ in (A.6) can be obtained by replacing $X_{S R^{*}}$ with $x_{S R^{*}}$ in (A.4) and (A.5). When $\Gamma_{R}<\Gamma_{D} \wedge\left(\theta_{1}-\Gamma_{D}\right)$ case, the outage probability of (A.6) can be written by (A.7), where $\varepsilon=\Gamma_{D} / \theta_{3}\left(\theta_{1}-\Gamma_{D}\right)$. On the other hand, when $\Gamma_{R} \geq \Gamma_{D} \wedge\left(\theta_{1}-\Gamma_{D}\right)$ case, the outage probability of (A.6) can be obtained by

$$
\begin{aligned}
P_{o, R^{*}}= & 1-\exp \left(-\frac{\Gamma_{R}}{\Omega_{S R^{*}} \theta_{3}}\right)+\frac{\Omega_{S Q} \Gamma_{R}}{\Omega_{S R^{*}} \theta_{2}+\Omega_{S Q} \Gamma_{R}} \\
& \times \exp \left\{-\left(\frac{\theta_{2}}{\Omega_{S Q} \Gamma_{R}}+\frac{1}{\Omega_{S R^{*}}}\right) \frac{\Gamma_{R}}{\theta_{3}}\right\} \quad, \Gamma_{R} \geq \Gamma_{D} /\left(\theta_{1}-\Gamma_{D}\right)
\end{aligned}
$$

\section{References}

[1] Y. Saito, Y. Kishiyama, A. Benjebbour, T. Nakamura, A. Li, and K. Higuchi, "Nonorthogonal multiple access (NOMA) for cellular future radio access", In: Proc. of Vehicular Technology Conf. (VTC Spring), pp.1-5, 2013.

[2] L. Dai, B. Wang, Y. Yuan, S. Han, C.-L. I, and Z. Wang, "Non-orthogonal multiple access for 5G: solutions, challenges, opportunities, and future research trends", IEEE Communications Magazine, Vol.53, No.9, pp.74-81, 2015.

[3] Z. Ding, Y. Liu, J. Choi, Q. Sun, M. Elkashlan, C.-L. I, and H. V. Poor, "Application of nonorthogonal multiple access in LTE and $5 \mathrm{G}$ networks", IEEE Communications Magazine, Vol.55, No.2, pp.185-191, 2017.

[4] W. Cai, C. Chen, L. Bai, Y. Jin, and J. Choi, "User selection and power allocation schemes for downlink NOMA systems with imperfect CSI", In: Proc. of Vehicular Technology Conf. (VTC-fall 2016), pp. 1-5, 2016.

[5] D. Wan, M. Wen, F. Ji, Y. Liu, and Y. Huang, "Cooperative NOMA systems with partial channel state information over Nakagami-m fading channels", IEEE Transactions on Communications, Vol.66, No.3, pp.947-958, 2017.

[6] Z. Ding, M. Peng, and H. V. Poor, "Cooperative non-orthogonal multiple access in 5G systems",
IEEE Communications Letters, Vol.19, No.8, pp.1462-1465, 2015.

[7] Y. Liu, Z. Ding, M. Elkashlan, and H. V. Poor, "Cooperative non-orthogonal multiple access with simultaneous wireless information and power transfer", IEEE Journal on Selected Areas in Communications, Vol.14, No.4, pp.938-953, 2016.

[8] N.-S. Kim, "Utilization of max-min user relay in cooperative NOMA systems", Journal of Telecommunications, Electronic and Computer Engineering, Vol. 10, No.1-4, pp.89-94, 2018.

[9] N.-S. Kim, "Performance of max-min user relay cooperative NOMA systems with imperfect CSI", International Journal of Intelligent Engineering and Systems, Vol.11, No.6, pp.148$155,2018$.

[10] Y. Xing, R. Chandramouli, S. Mangold, and S.S. Nandagopalan, "Dynamic spectrum access in open spectrum wireless networks", IEEE Journal on Selected Areas in Communications, Vol.24, No.3, pp.626-637, 2006.

[11] T. M. C. Chu, H. Phan, and H.-J. Zepernick, "Opportunistic spectrum access for cognitive amplify-and-forward relay networks", In: Proc. of Vehicular Technology Conf. (VTC Spring), pp.1-5, 2013.

[12] M. Poreddy, T. M. C. Chu, and H.-J. Zepernick, "On outage probability of cooperative cognitive radio networks over $\kappa-\mu$ shadowed fading", In: Proc. of Wireless Communications and Networking Conf. (WCNC), pp.1-6, 2017.

[13] X. Kang, Y.-C. Liang, and A. Nallanathan, "Optimal power allocation for fading channels in cognitive radio networks under transmit and interference power constraints", In: Proc. of IEEE International Conf. On Communications, pp.3568-3572, 2008.

[14] Y. Liu, Z. Ding, M. Elkashlan, and J. Yuan, "Nonorthogonal multiple access in large-scale underlay cognitive radio networks", IEEE Transactions on Vehicular Technology, Vol.65, No.12, pp.10152-10157, 2016.

[15] L. Lv, J. Chen, and Q. Ni, "Cooperative nonorthogonal multiple access in cognitive radio", IEEE Communications Letters, Vol.20, No.10, pp.2059-2062, 2016.

[16] M. Zeng, G. I. Tsiropoulos, O.A. Dobre, and M. H. Ahmed, "Power allocation for cognitive radio networks employing non-orthogonal multiple access", In: Proc. of IEEE Global Communications Conf. (GLOBECOM), pp.1-5, 2016. 
[17] T. M. C. Chu and H.-J. Zepernick, "Nonorthogonal multiple access for DF cognitive cooperative radio networks", In: Proc. of International Conf. on Communications Workshop (ICC Workshops), pp.1-6, 2018.
[18] L. Xu, Y. Zhou, P. Wang, and W. Liu, "Maxmin resource allocation for video transmission in NOMA-based cognitive wireless networks", IEEE Transactions on Communications, Vol.66, No.11, pp.5804-5813, 2018. 